\title{
三江平原湿地小叶章生产力模拟模型
}

\author{
张文菊 ${ }^{12}$ 童成立 ${ }^{1}$ 刘守龙 ${ }^{12}$ 宋长春 $^{3}$ 吴金水 $^{1 *}$ \\ (1 中国科学院亚热带农业生态研究所亚热带农业生态重点实验室, 长沙 410125) \\ （2 华中农业大学资源与环境学院, 武汉 430070) (3 中国科学院东北地理与农业生态研究所, 长春 130012)
}

摘 要 利用气象台站的常规观测资料, 依据植物生长模拟理论, 以 $\mathrm{d}$ 为步长 建立了湿地小叶章( Deyeuxia angustifolia) 植被生产力动态模拟模型。该模型包括 3 个子模块 : 1) 光合作用与呼吸作用 2)干物质积累 3)同化物分配 ;主 要考虑了温度和积水因子对植物生长的影响。并利用实测资料对该模型进行了检验，结果表明: 小叶章地上活体、 枯落物、茎、叶各器官枯落物的模拟值与实测值之间均呈极显著的线性相关 $\left(R^{2}\right.$ 分别为 $0.98 、 0.99 、 0.99$ 和 0.92$)$ 。 在相邻区域的检验结果也表明，季节性积水沼泽化草甸小叶章的地上生物量明显高于常年积水沼泽。两类湿地小 叶章地上生物量的模拟值与实测值之间均呈极显著线性相关 ( $R^{2}$ 分别为 0.66 和 0.79$)$ 。相近区域长期定位观测点 连续 2 年的模拟结果与实测值之间也具有极显著的线性相关 $\left(R^{2}\right.$ 分别为 0.97 和 0.76$)$ 。

关键词 湿地生态系统 三江平原 生物量 模拟模型

\section{A MODEL TO SIMULATE NET PRIMARY PRODUCTION OF DEYEUXIA ANGUSTIFOLIA IN WETLANDS IN SANJIANG PLAIN , CHINA}

\author{
ZHANG Wen-Ju ${ }^{12}$ TONG Cheng-Li ${ }^{1} \quad$ LIU Shou-Long ${ }^{12}$ SONG Chang-Chun ${ }^{3}$ and WU Jin-Shui ${ }^{1}$ * \\ (1 Key Laboratory of Subtropical Agro-Ecology, Institute of Subtropical Agriculture , Chinese Academy of Sciences , Changsha 410125 , China) \\ ( 2 College of Resource and Environment, Huazhong Agricultural University, Wuhan 430070 , China) \\ (3 Northeast Institute of Geography and Agricultural Ecology , Chinese Academy of Sciences, Changchun 130012, China)
}

\begin{abstract}
Background and Aims Carbon sequestration in ecosystems is determined largely by primary production of ecosystems. The application of long-term observation of vegetation biomass limits ecological and global change research. Therefore, it is necessary to apply simulation models to estimate an ecosystem's input of organic carbon and forecast the impact of climate change.

Methods Based on the principles of plant physiology , a simulation model was developed to simulate primary production of Deyeuxia angustifolia in wetland ecosystems. It is driven by conventional parameters observed from meteorological stations. This model consists of three main function modules : photosynthesis-respiration , accumulation of assimilated matter and distribution of assimilated matter. The effects of temperature, waterlogging and increased concentration of atmosphere $\mathrm{CO}_{2}$ on the growth of $D$. angustifolia were taken into account in this model. The model was validated by comparing observed and simulated data of aboveground living and litter biomass of $D$. angustifolia .
\end{abstract}

Key Results The dynamics of aboveground living biomass, total litter, stem litter and leaf litter of $D$. angustifolia were simulated correctly. The liner correlation between simulated and observed values was highly significant.

Conclusions Validation results from an adjacent area and a field experiment station also showed that the simulated values were significantly correlated with observed values in seasonally and permanently waterlogged $D$. angustifolia ecosystems. Daily changes of primary production, biomass and liter of $D$. angustifolia in wetland ecosystems could be effectively simulated by this model.

Key words Wetland ecosystem , Sanjiang Plain , Biomass , Simulating model

湿地不仅是巨大的天然物种库，而且具有独特 的环境功能和生态效益。自 1995 年联合国气候变 化框架公约 (UNFCCC) 将湿地保护列为一项增加碳
汇功能的措施之后, 湿地生态系统植被固碳潜力的 研究成为全球碳循环和全球变化的重要研究内容 (Richert et al ., 2000 ; Sorrell et al ., 2000 ;Camill et 
al . 2001)。湿地植被对大气中 $\mathrm{CO}_{2}$ 的固定是湿地 生态系统碳循环的重要过程。以往研究中, 生物量 监测是了解湿地植被生产力状况的主要途径, 但其 缺点是难以大范围、全方面 (包括各种植被类型、不 同环境条件)的实施。计算机模型使得大范围的生 物量和净初级生产力 $(N P P)$ 的估算成为可能 (Melillo et al . , 1993) ,并已逐步成为定量描述生态系统植 被生产力特征及其对全球变化响应方面研究的一项 有效手段(Woodward et al ，１995 ;周广胜和张新时， 1996 㹈梅等，1997; 延晓冬等，2000)。最早应用于 估算生态系统 NPP 的模型是统计模型 (气候模型), 它能够直接反映气候因子与 $N P P$ 之间的关系。我 国一些学者曾采用统计模型对一些湿地植物的季节 生长动态进行了研究(马克平等, 1993 ; 倪红伟等, 1999 , 李英年等, 2004)。但由于地理位置、植被类 型、气候条件以及方法等缺乏统一标准, 这些统计模 型的普遍性和适用性受到了限制，而且模型过于简 单, 难以反映植物的生长发育过程 (郝永萍等, 1998)。模拟模型则从植物生长的生理学角度出发, 不仅可以在一定程度上克服统计模型的缺陷,而且 还可以考虑众多环境因子对群体植物生长的影响， 进行逐日乃至逐时的动态模拟。在气候变暖和大气 $\mathrm{CO}_{2}$ 浓度升高的背景下, 模拟模型的建立和应用显 得尤为重要(Rasse et al.，2002;杨兆芳等，2005)。 本研究基于光、温、水等环境因子的影响和植被自身 的光合、呼吸作用，从生理生态过程建立了典型湿地 植被小叶章的生长动态模拟模型, 以期为湿地植被 生产力和固碳潜力研究以及整个湿地生态系统的碳 源、汇功能评价提供科学依据。

\section{1 研究区概况}

三江平原 $\left(129^{\circ} 11^{\prime} \sim 135^{\circ} 05^{\prime} \mathrm{N}, 43^{\circ} 49^{\prime} \sim 48^{\circ} 27^{\prime}\right.$ E)位于黑龙江省东北部, 是由黑龙江、松花江和乌 苏里江冲积形成的凹低平原。该地属于暖温带湿润 大陆性季风气候, 年均气温 $1.9 \sim 3.6{ }^{\circ} \mathrm{C}$, 年降水 $500 \sim 650 \mathrm{~mm}$ 。因受季风影响，全年降水量的75\% $85 \%$ 集中分布于夏、秋两季, 这使得该地区的一些洼 地极易形成地表积水; 而季节性冻土结冻的时间长 达 6 个月之久 冻层深至地下 $160 \sim 180 \mathrm{~cm}$ 。冻层形 成的隔水板使地表积累的雪水不能下渗, 为沼泽的 发育提供了良好的水文条件。由河漫滩、阶地和山 前倾斜平原组成的起伏较小的地貌条件是该地区沼 泽形成的基础。该地区湿地类型较简单, 均为季节 性或常年积水的草丛沼泽, 其典型的优势植被主要 有小叶章 (Deyeuxia angustifolia) 和一些苔草群落, 如 毛果苔草 (Carex lasiocapa)、漂笺苔草 ( C. pseudocuraica)、乌拉苔草 $(C$. meyeriana $)$ 等。

\section{2 研究资料与方法}

\section{1 资料来源}

本模型利用气象台站常规的监测数据,包括逐 日的太阳辐射、气温、降水及日照时数等，建立湿地 典型植被小叶章的生物量动态模拟模型。在没有太 阳辐射观测值的区域, 则采用日照时数观测结果模 拟计算太阳辐射,计算方法见童成立等 (2005)。模 型的其它驱动因子，包括大气 $\mathrm{CO}_{2}$ 浓度、经纬度、湿 地类型以及积水状况等。模型检验所用气象和生物 量数据来源见表 1 。

表 1 模型检验实测数据来源

Table 1 Observed data resource for the model validation

\begin{tabular}{|c|c|c|c|c|}
\hline $\begin{array}{c}\text { 地点 } \\
\text { Site }\end{array}$ & $\begin{array}{l}\text { 时间 } \\
\text { Time }\end{array}$ & $\begin{array}{c}\text { 经纬度 } \\
\text { Geographic position }\end{array}$ & $\begin{array}{l}\text { 数据类型 } \\
\text { Data type }\end{array}$ & $\begin{array}{l}\text { 数据来源 } \\
\text { Data resource }\end{array}$ \\
\hline $\begin{array}{l}\text { 黑龙江宝清原种场 } \\
\text { Original breed farm in Baoqing, } \\
\text { Heilongjiang Province }\end{array}$ & $1988 \sim 1989$ & $\begin{array}{l}46^{\circ} 19^{\prime} \mathrm{N} \\
132^{\circ} 11^{\prime} \mathrm{E}\end{array}$ & $\begin{array}{l}\text { 气象数据 } \\
\text { Meteorological data } \\
\text { 生物量 Biomass }\end{array}$ & $\begin{array}{l}\text { 国家气象站(宝清站) } \\
\text { National meteorological station（Baoqing) } \\
\text { 马克平，1993；马克平等，1993 }\end{array}$ \\
\hline $\begin{array}{l}\text { 黑龙江宝清七星河乡 } \\
\text { Qixinghe , Baoqing, } \\
\text { Heilongjiang Province }\end{array}$ & $1992 \sim 1993$ & $\begin{array}{l}46^{\circ} 45^{\prime} \mathrm{N} \\
132^{\circ} 05^{\prime} \mathrm{E}\end{array}$ & $\begin{array}{l}\text { 气象数据 } \\
\text { Meteorological data } \\
\text { 生物量 Biomass }\end{array}$ & $\begin{array}{l}\text { 国家气象站(宝清站) } \\
\text { National meteorological station (Baoqing) } \\
\text { 倪红伟等，1999 }\end{array}$ \\
\hline \multirow[t]{2}{*}{$\begin{array}{l}\text { 三江平原沼泽湿地生态试验站 } \\
\text { Swamp ecological experiment } \\
\text { station in Sanjiang Plain }\end{array}$} & $2003 \sim 2004$ & $\begin{array}{l}47^{\circ} 35^{\prime} \mathrm{N} \\
133^{\circ} 17^{\prime} \mathrm{E}\end{array}$ & $\begin{array}{l}\text { 气象数据 } \\
\text { Meteorological data }\end{array}$ & $\begin{array}{l}\text { 三江平原沼泽湿地生态试验站 } \\
\text { Swamp ecological experiment station in Sanjiang Plain }\end{array}$ \\
\hline & & & 生物量 Biomass & $\begin{array}{l}\text { 三江平原沼泽湿地生态试验站 } \\
\text { Swamp ecological experiment station in Sanjiang Plain }\end{array}$ \\
\hline
\end{tabular}




\section{2 模型的建立}

模型由 3 个模块组成, 即 : 1) 光合作用和呼吸作 用 2)干物质积累 3) 同化物分配。模拟时段为植被 生长开始到生长结束, 模拟步长为 $\mathrm{d}$ 。由于湿地植 被处于自然生长状态, 其生长和死亡采用温度控制, 抽穗结实根据生长期判定。本模型假设湿地植物的 生长发育不受养分条件的限制，无病虫害的影响。

\subsection{1 光合作用和呼吸作用子模块}

\subsubsection{1 光合作用}

综合光、温、水和植被自身对光合作用的影响， 采用下列形式计算实际条件下自生长开始后第 $\mathrm{i}$ 天 的光合作用强度 $P_{(i)}\left(\mathrm{g} \mathrm{m}^{-2} \mathrm{~d}^{-1}\right)$ :

$$
P_{(i)}=\varepsilon \times P g_{(i)} \times A_{(i)}
$$

式中 : $P g_{(i)}$ 为适宜的热量、水分保证和实际光照条 件下的光合速率 $\left(\mathrm{g} \mathrm{CO}_{2} \mathrm{~m}^{-2} \mathrm{~d}^{-1}\right) ; A_{(i)}$ 为气象和环 境因子影响函数: $\varepsilon$ 为 $\mathrm{CO}_{2}$ 与碳水化合物的转化率， $\varepsilon=\left(\mathrm{CO}_{2}\right) /\left(\mathrm{CH}_{2} \mathrm{O}\right)=0.68$ 。 $P g_{(i)}$ 采用最常见的叶光 合速率公式 Monsi-Saeki (米氏) 方程计算 (France \& Thornley, 1991) :

$$
P g_{(i)}=\frac{\alpha I_{1(i)} \times P_{\max (\mathrm{i})}}{\alpha I_{1(i)}+P_{\max (\mathrm{i})}}
$$

投射在叶片上的光通量密度 $I_{1(i)}\left(\mathrm{J} \mathrm{m}^{-2}\right)$ 通过 光能在冠层分布公式计算：

$$
I_{1(i)}=\left(\frac{k I_{0(i)}}{1-m}\right) \times e^{-k L A I_{(i)}}
$$

式中: $I_{0(i)}$ 为冠层之上的光通量密度; $k$ 为消光系 数; $m$ 为叶片透射率; $a$ 为光合作用响应曲线的初始 斜率 $\left(\mathrm{g} \mathrm{CO}_{2} \mathrm{~J}^{-1}\right) ; L A I_{(i)}$ 为叶面积指数。

假定全天 $P_{\max (i)}$ (最大光合速率) 是外界 $\mathrm{CO}_{2}$ 浓 度的一个函数, $P_{\max }(i)$ 与外界 $\mathrm{CO}_{2}$ 浓度的依赖性以 下式表达(France \& Thornley, 1991)：

$$
P_{\max (i)}=\tau \times C_{\mathrm{CO}_{2}} \times 1.83
$$

式中 : $\tau$ 是 $\mathrm{CO}_{2}$ 的传导系数 其生物学意义是光饱和 时每增加单位 $\mathrm{CO}_{2}$ 浓度所增加的光合速率(喻梅等, 1997); $C_{\mathrm{CO}_{2}}$ 为大气 $\mathrm{CO}_{2}$ 浓度 $\left(\mathrm{g} \mathrm{CO}_{2} \mathrm{~m}^{-3}\right), 1.83$ 为大 气 $\mathrm{CO}_{2}$ 浓度 $\left(\mu \mathrm{L}^{-1}\right)$ 与 $\mathrm{C}_{\mathrm{CO}_{2}}\left(\mathrm{~g} \mathrm{CO}_{2} \mathrm{~m}^{-3}\right)$ 之间的转 换系数。

通常认为全天光通量密度近似等于光合有效辐 射, 即 :

$$
I_{0(i)}=P A R_{(i)}
$$

式中 : $P A R_{(i)}$ 为光合有效辐射 $\left(\mathrm{J} \mathrm{m}^{-2} \mathrm{~d}^{-1}\right)$, 由下式 计算:

$$
P A R_{(i)}=C_{Q} \times Q_{(i)}
$$

式中: $C_{Q}$ 为太阳总辐射对光合有效辐射的转换系 数, 它随太阳高度、光质、云量等因素变化而变化 (周 允华, 1988), $Q_{(i)}$ 为太阳总辐射 $\left(\mathrm{J} \mathrm{m}^{-2} \mathrm{~d}^{-1}\right)$ 。

\subsubsection{2 呼吸作用}

呼吸作用 $\left(R_{(i)}, \mathrm{g} \quad \mathrm{m}^{-2} \cdot \mathrm{d}^{-1}\right)$ 分为生长呼吸 (Growth respiration, $G R_{(i)}$ ) 和维持呼吸 (Maintenance respiration, $\left.M R_{(i)}\right)$ 两部分。

生长呼吸与光合速率有关 对温度反应不敏感; 维持呼吸与植株干重及温度有关, 同时需考虑地上、 地下部分呼吸特性的差异。

$$
\begin{aligned}
& R_{(i)}=G R_{(i)}+M R_{(i)} \\
& G R_{(i)}=C g \times P_{(i)} \\
& M R_{(i)}=M R_{(T(i))} \times Q_{10}\left(T_{(i)}-T_{0}\right) / 10 \\
& M R_{(T(i))}=k_{1} \quad M_{1}+k_{2} \quad M_{2}
\end{aligned}
$$

式中 : $C_{g}$ 为生长呼吸系数; $k_{1}$ 和 $k_{2}$ 分别为地上、地 下部分的维持呼吸系数; $M_{1}$ 和 $M_{2}$ 分别为当前植被 地上、地下生物量干重 $\left(\mathrm{g} \mathrm{m}^{-2}\right) ; M R_{(T(i))}$ 为最适气 温 $\left(25{ }^{\circ} \mathrm{C}\right)$ 时的维持呼吸; $T_{(i)}$ 为日平均气温 $\left({ }^{\circ} \mathrm{C}\right)$; $Q_{10}$ 为呼吸作用的温度系数。

表 2 湿地植被生产力模拟模型主要参数来源

Table 2 Parameters for simulating model of vegetation primary productivity in wetlands

消光系数 Extinction coefficient $(k)$

叶片透射率 Leaf transmissivity $(m)$

光合作用响应曲线初始斜率 Initial slope of photosynthesis response curve $(\alpha)$

$\mathrm{CO}_{2}$ 传导系数 $\mathrm{CO}_{2}$ Conductivity coefficient $(\tau)$

地上部分维持呼吸系 Aboveground maintenance respiration coefficient $\left(k_{1}\right)$

地下部分维持呼吸系数 Underground maintenance respiration coefficient $\left(k_{2}\right)$

生长呼吸系数 Growth Respiration coefficient $(C g)$

呼吸作用的温度系数 Temperature coefficient $\left(Q_{10}\right)$

总辐射对光合有效辐射的转换系数 Coefficient of total radiation to $P A R\left(C_{Q}\right)$
数值 Value

0.5

0.12

$12 \times 10^{-6}$

0.002

0.015

0.022

0.30

2.0

0.5
来源与说明 References and note 刘以连和丑自明, 1994 刘以连和丑自明, 1994 France \& Thornley , 1991 France \& Thornley , 1991 de Penning Vries \& van Laar , 1986 de Penning Vries \& van Laar , 1986 de Penning Vries \& van Laar , 1986 黄耀等, 2005 周允华 ,1988 


\subsection{2 干物质积累子模块}

冠层每日的干物质生产量 $\left(\Delta W_{(i)}, \mathrm{g} \mathrm{m}^{-2} \mathrm{~d}^{-1}\right)$ 为冠层日同化量扣除呼吸消耗：

$$
\Delta W_{(i)}=P_{(i)}-R_{(i)}
$$

植被干物质积累 $W_{(i)}\left(\mathrm{g} \mathrm{m}^{-2}\right)$ 为 $\Delta W_{(i)}$ 随生长 进程的积分：

$$
W_{(i)}=\int_{0}^{t} \Delta W_{(i)} \times d t
$$

式中 : $W_{(i)}$ 的初始值取 $0, t$ 为植被生长进程 $(\mathrm{d})$ 。

采用每日冠层的净同化量及叶片分配指数来计 算日叶片干物质重 $\left(\Delta W L_{(i)}, \mathrm{g} \mathrm{m}^{-2}\right)$, 通过总叶片干 物质重与叶立枯量之差 $\left(\Delta L L_{(i)}\right)$ 和比叶面积 ( $S L A$, $\left.\mathrm{m}^{2} \mathrm{~g}^{-1}\right)$ 模拟叶面积指数动态 $\left(L A I_{(i)}\right)$ 。

$$
\begin{aligned}
& \Delta W L_{(i)}=C L_{(i)} \times \Delta W_{(i)} \\
& W L_{(i)}=W L_{(i-1)}+\left(\Delta W L_{(i)}-\Delta L L_{(i)}\right) \\
& L A I_{(i)}=W L_{(i)} / S L A
\end{aligned}
$$

式中 : $C L_{(i)}$ 为每日同化物向叶片的分配比例, $W L_{(i)}$ 为叶片干物质重。

\subsection{3 同化物分配}

\subsubsection{1 同化物分配}

干物质形成后分别分配到不同的器官。三江平 原湿地小叶章的器官主要包括根、茎、叶、穗 $(J)$ 。 各功能器官干物质日生产量 $\Delta W J_{(i)}\left(\mathrm{g} \mathrm{m}^{-2} \mathrm{~d}^{-1}\right)$ 和 累积量 $W J_{(i)}\left(\mathrm{g} \mathrm{m}^{-2}\right)$ :

$$
\begin{aligned}
& \Delta W L_{(i)}=C J_{(i)} \times \Delta W_{(i)} \\
& W J_{(i)}=\int_{0}^{\mathrm{t}} \Delta W J_{(i)} \times d t
\end{aligned}
$$

式中: $C_{J(i)}$ 为净光合产物向各功能器官的分配系 数。在不同的发育阶段, 各器官也对应不同的分配 比例。小叶章各器官的分配系数主要参考马克平 (1995)和倪宏伟 (1996) 的试验结果进行估算。对于 多年生的湿地植物小叶章, 自抽穗结实后, 茎部分的 营养物质会转移到根。因此, 根与茎的干物质重以 (19)、(20)式模拟。

\subsubsection{2 茎营养物质转移到根}

对于多年生的湿地植物, 自抽穗结实后, 苳部分 的营养物质会向根转移。因此, 本模型中考虑了部 分茎的营养物质转移到根 $\left(\Delta W S R_{(i)}, \mathrm{g} \mathrm{m}^{-2}\right)$ :

$$
\Delta W S R_{(i)}=\lambda \times W S_{(i)}
$$

式中 : $\lambda$ 为营养物质的转移系数。则根与茎的干物 质重 $\left(W R_{(i)}, W S_{(i)}, \mathrm{g} \mathrm{m}^{-2}\right)$ 分别为:

$$
\begin{aligned}
& W R_{(i)}=\Delta W S R_{(i)}+\Delta W R_{(i)} \\
& W S_{(i)}=W S_{(i)} \times(1-\lambda)
\end{aligned}
$$

\subsubsection{3 枯落物的形成}

各功能器官的日枯落量 $\left(\Delta L J_{(i)}, \mathrm{g} \mathrm{m}^{-2}\right)$ :

$$
\begin{aligned}
& \Delta L J_{(i)}=\beta J_{(i)} \times W J_{(i)} \\
& L J_{(i)}=\int_{0}^{t} \Delta L J_{(i)} \times d t
\end{aligned}
$$

式中: $\beta J_{(i)}$ 为各器官日枯落物占器官总重的比例, $L_{J(i)}$ 为枯落物量。

\subsection{4 气象和环境因子函数 $\mathrm{A}$ 的计算}

从影响湿地植被生产力的环境因子中, 选取气 温和湿度因子。环境影响因子 $A_{(i)}$ 为：

$$
A_{(i)}=A_{T(i)} \times A_{W(i)}
$$

温度因子的影响如下式：

$$
A_{T(i)}=\exp \left[a_{1} \times\left(\frac{T_{i}-T_{0}^{2}}{10}\right)\right]\left(0<A_{T(i)} \leq 1\right)
$$

式中 : $T_{i}$ 为白天平均气温 $\left({ }^{\circ} \mathrm{C}\right) ; T_{0}$ 为植物生长光合 速率最适气温, 参考刘以连和丑自明 (1994) 研究, 最 适气温在拔节前取 $21{ }^{\circ} \mathrm{C}$, 拔节后取 $25^{\circ} \mathrm{C}$ 。

$$
a_{1}= \begin{cases}-1 & \text { 当 } T_{i}<T_{0} \\ -2 & \text { 当 } T_{i}>T_{0}\end{cases}
$$

模型中，我们没有全面地考虑水分亏缺对植物 生长的影响。但湿地植物对水分的敏感性仍存在一 定差异, 不同程度的积水对同一类型的植物存在影 响(刘振乾等，2002)。因此，同湿地类型的植物对 水分的敏感性 $A_{W(i)}$ 为经验性参数, 主要参考刘振乾 等(2002)研究进行校正。

\section{3 结果与分析}

图 1 为三江平原草甸生态系统小叶章地上生物 量动态模拟结果。小叶章地上生物量的季节动态呈 单峰型, 在 7 月下旬达到最大值 (杨永兴等, 2002)。 生长高峰期过后, 随着秋季的来临, 气温的降低, 小 叶章的光合能力减弱, 渐趋衰老, 枯落物的量增加, 营养物质溶失及向地下根系转移过程日益旺盛, 导 致地上活体生物量日趋减少, 直至 10 月中旬停止生 长。这种单峰型生长曲线与三江平原地区温带季风 性气候的干、湿季节明显, 雨、热同季的特点相适应 (倪红伟等, 1998)。小叶章枯落物主要有叶、茎和 穗组成, 枯落物形成早期以叶居多, 随着生长期的推 移, 茳和穗枯落物出现, 但穗所占比例很小。相关性 分析表明 模型模拟的小叶章地上活体生物量、地上 枯落量及茎和叶枯落量的模拟值与实测值之间具有 极显著的线性相关关系(图 2)，相关系数 $R^{2}$ 分别为 $0.98 、 0.99 、 0.99$ 和 0.92 。

湿地的水分状况决定其建群种植被类型 (优势 植被)。湿地由外向内, 由于地势逐渐降低, 积水条 件增强, 建群种植物依次为小叶章群落、小叶章 + 毛 果苔草群落、毛果苔草 + 漂筏苔草 + 芦苇 $($ Phrag- 
mites communis) 群落。中间积水较深的则为挺水植 物或水生植物。地表积水明显抑制小叶章生长(刘 振乾等，2002)。沼泽中小叶章群落地上生物量明 显低于沼泽化草甸 (图 3)。本模型对沼泽化草甸和 沼泽中小叶章群落地上生物量模拟值与实测值之间 均呈极显著线性相关, $R^{2}$ 分别为 0.66 和 0.79 (图
4）表明建立的植被生物量模型能较好地模拟三江 平原典型湿地植被小叶章群落的地上生物量动态。 长期定位试验观测数据 (2003 2004 年) 的检验结果 也表明 模型对湿地小叶章的生物量动态的模拟值 与实测值之间呈极显著的线性相关关系, 相关系数 $R^{2}$ 分别为 0.97 和 0.76 (图 5 , 图 6)。

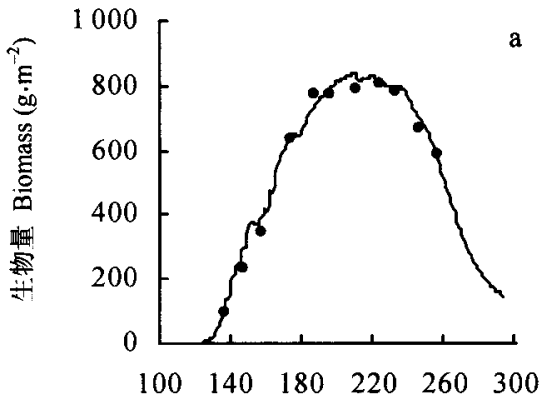

生长时间 Time (d)

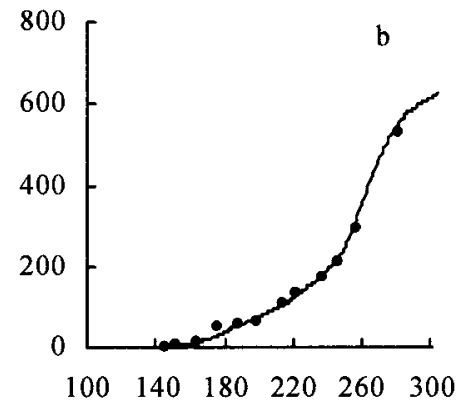

生长时间 Time (d)

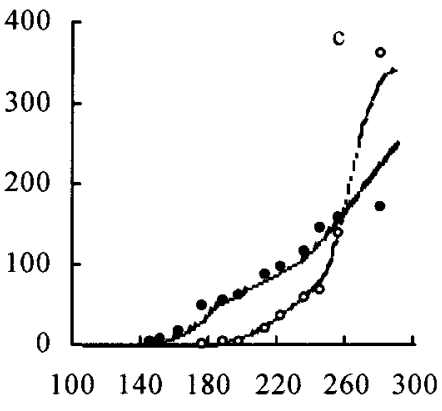

生长时间 Time (d)

图 1 三江平原草甸生态系统植被小叶章地上生物量动态模拟

Fig.1 Simulating aboveground biomass of typical wetland vegetation (Deyeuxia angustifolia) in the meadow of Sanjiang Plain

a 地上活体生物量 Aboveground living biomass b 地上枯落物 Aboveground litter biomass c 地上茎、叶枯落物 (实线为模拟值, 符点为实测值) Aboveground litter biomass from stem and leaf (Real line for the simulated data, symbol for the observed data) - $\bigcirc$ - : 荭枯落物 Stem litter - - : 叶 枯落物 Leaf litter 实测值引自马克平(1993)和马克平等(1993) Observed data from Ma (1993), Ma et al. (1993)

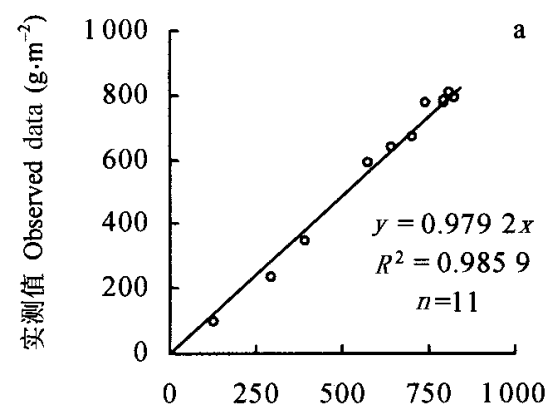

模拟值 Simulated data $\left(\mathrm{g} \cdot \mathrm{m}^{-2}\right)$

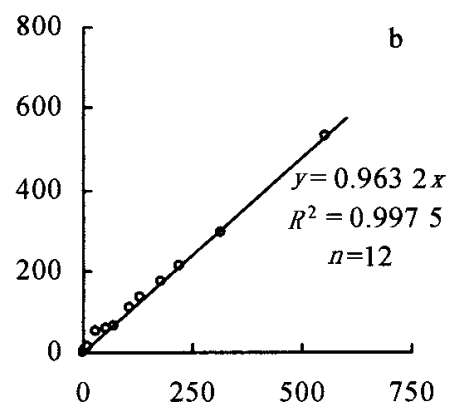

模拟值 Simulated data $\left(\mathrm{g} \cdot \mathrm{m}^{-2}\right)$

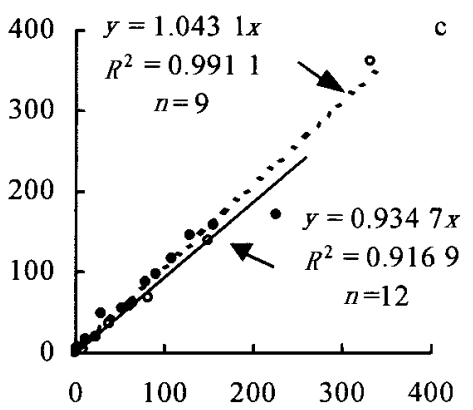

模拟值 Simulated data $\left(\mathrm{g} \cdot \mathrm{m}^{-2}\right)$

图 2 三江平原草甸生态系统植被小叶章模拟值与实测值的拟合关系

Fig.2 Correlation between simulated and observed of Deyeuxia angustifolia in the meadow of Sanjiang Plain

a 地上活体生物量 Aboveground living biomass b 地上枯落物 Aboveground litter biomass c 地上茎、叶枯落物 Aboveground litter biomass from stem and leaf $-\bigcirc-$ : 茎枯落物 Stem litter

- : 叶枯落物 Leaf litter 实测值引自马克平 (1993)和马克平等 (1993) Observed data from Ma (1993) , Ma et al. (1993)

\section{4 讨 论}

本模型利用常规的气象指标为驱动因子，综合 考虑了植物的光合、呼吸作用等过程，模拟湿地典型 植被小叶章的生物量动态。与实测值的比较说明模 拟结果符合自然条件下小叶章的生长规律，可以用
来预测湿地小叶章的生产力、干物质的积累量及其 动态变化, 以及湿地生态系统的有机物输入量动态, 同时也能为湿地沉积物基质碳循环模型的开发和研 制提供必要的科学依据和参数。一般情况下, 植物 生长不仅受气象条件和作物本身的遗传因素的影 响，还受到水分、养分 (氮、磷或其它矿质养分) 短 

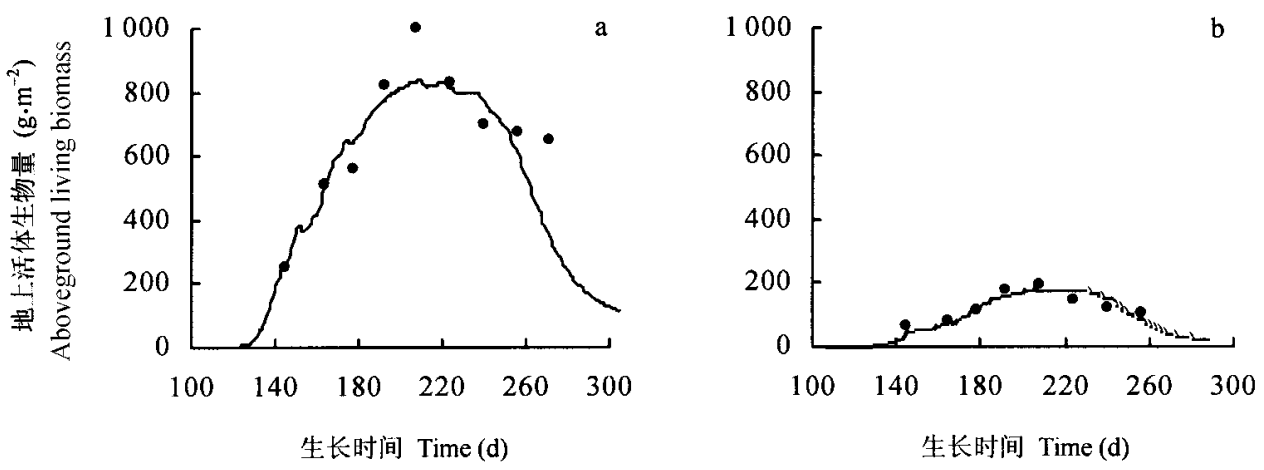

图 3 三江平原典型湿地小叶章群落地上活体生物量动态模拟

Fig.3 Simulating abovegroundliving biomass of Deyeuxia angustifolia in two typical wetlands in Sanjiang Plain

$\mathrm{a}$ 季节性积水沼泽 Seasonally waterlogged $\mathrm{b}$ 常年积水沼泽 Permanently waterlogged 实线为模拟值, 符点为实测值 Real line for the simulated data , symbol for the observed data 实测值引自倪红伟等(1999) Observed data from Ni et al. (1999)
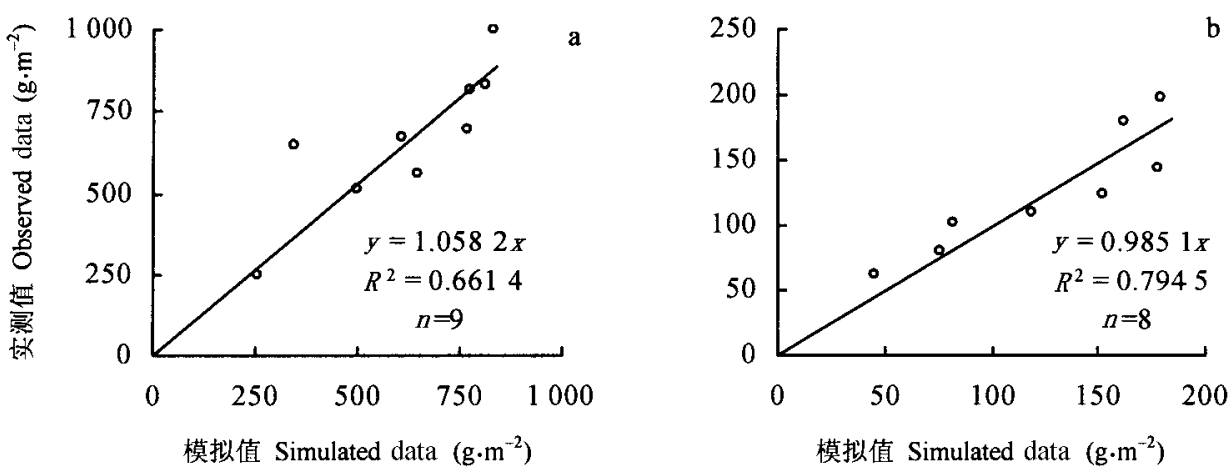

模拟值 Simulated data $\left(\mathrm{g} \cdot \mathrm{m}^{-2}\right)$

图 4 湿地生态系统植被小叶章地上生物量模拟值与实测值的拟合关系

Fig.4 Correlation between simulated and observed data of Deyeuxia angustifolia in two typical wetlands in Sanjiang Plain $\mathrm{a}$ 季节性积水沼泽 Seasonally waterlogged $\mathrm{b}$ 常年积水沼泽 Permanently waterlogged 实测值引自倪红伟等 (1999) Observed data from Ni et al. (1999)
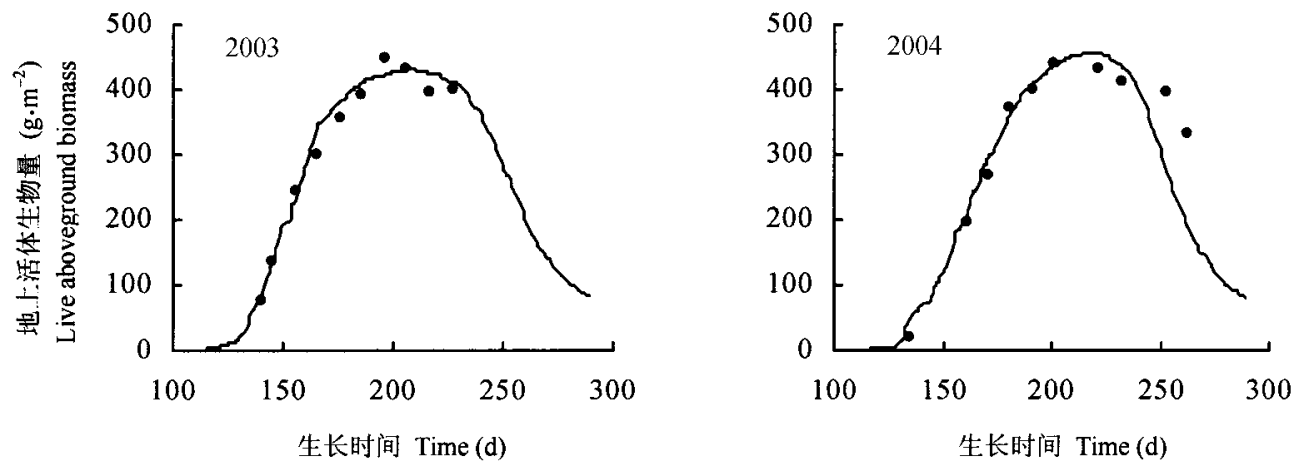

图 5 三江平原沼泽化草甸小叶章群落地上活体生物量动态模拟

Fig.5 Simulating aboveground living biomass of Deyeuxia angustifolia in the marshy meadow in Sanjiang Plain 实线为模拟值，符点为实测值 Real line for the simulated data， symbol for the observed data 


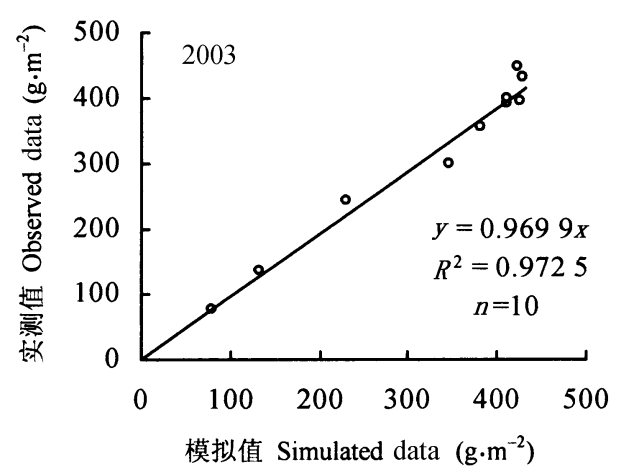

图 6 三江平原沼泽化草甸小叶章地上生物量模拟值与实测值的拟合关系

Fig.6 Correlation between simulated and observed data of Deyeuxia angustifolia in the marshy meadow in Sanjiang Plain

缺的制约，以及病虫害的影响 (de Penning Vries \& van Laar , 1986)。我们建模时考虑了主要气象因子和积 水程度的影响, 没有考虑养分亏缺和病虫害的影响。 尽管就整个地质大循环来说, 自然条件下的湿地养 分是基本平衡的 (Shukla, 1998)。大多数的湿地植 被生长模型都没有考虑养分亏缺对植物生长的影响 (Shukla， 1998 ;Sturtevant，1998;曲颖等，2004)。但 不同生长季节湿地植物对养分如 $\mathrm{N}$ 素的选择性吸 收可能限制其生长, 而且不同湿地植物对 $\mathrm{NO}_{3}-\mathrm{N}$ 或 $\mathrm{NH}_{4}-\mathrm{N}$ 的吸收能力还受积水环境的重要影响 (孙雪 利等, 2000)。大气 $\mathrm{CO}_{2}$ 浓度升高对植物生长具有 施肥效应” 本模型中我们引入 $\mathrm{CO}_{2}$ 传导系数表达 最大光合速率对 $\mathrm{CO}_{2}$ 浓度的依赖性。喻梅等 (1997) 认为传导系数与 $\mathrm{CO}_{2}$ 浓度有关,一些研究者则采用 校正因子来描述大气 $\mathrm{CO}_{2}$ 浓度升高对光合作用的影 响(Zhang et al.,2002 ;黄耀等, 2005)。

本模型是基于太阳辐射、温度、水分等因子建立 起来的植物生理生态动态模拟模型, 是湿地生态系 统碳循环模型的一个子模型, 主要着重描述植被与 大气界面之间的碳交换。由于实测资料的限制, 本 文只对小叶章的地上各器官的生物量动态进行了模 拟，一些参数如同化物的分配系数、水分影响因子等 具有一定的经验性, 需要更多的实测资料和试验来 进行验证。另外,不同区域湿地的气候存在较大差 异,由此产生不同区域湿地植被种群多样性和复杂 性对模拟模型提出了更高的要求。模型的适应性和 普遍性仍有待进一步验证。

\section{参 考 文 献}

Camill P, Lynch JA, Clark JS, Adams JB, Jordan B (2001).

Changes in biomass, aboveground net primary production, and peat accumulation following permafrost thaw in the boreal peat- lands of Manitoba, Canada. Ecosystems, 4, 461-478.

de Penning Vries FWT, van Laar HH (Translated by Wang FT (王 馥棠), Wang SL (王石立), Liu SZ (刘树泽), Qi LF (元来 福)) (1986). Simulation of Plant Growth and Crop Production (in English). Science Press, Beijing, 1-32. (in Chinese)

France J, Thornley JHM (Translated by Jin ZQ (金之庆), Gao LZ (高亮之)) (1991). Mathematical Models in Agriculture: a Quantitative Approach to Problems in Agriculture and Related Sciences (in English). China Agricuture Press, Beijing, 142 - 173. (in Chinese)

Hao YP (郝永萍), Chen YF (陈育峰), Zhang XY (张兴有) (1998). Progresses in estimation of net primary productivity and its responses to climate change. Advance in Earth Sciences (地球 科学进展), 13,564-571. (in Chinese with English abstract) Huang Y (黄耀), Yang ZF (杨兆芳), Yu YQ (于永强), Jiang J (姜军), Sun WJ (孙文娟) (2005). Simulating net primary production of rice and wheat crops: model establishment. Environmental Science (环境科学), 26(2), 11-15. (in Chinese with English abstract)

Li YN (李英年), Wang QX (王勤学), Gu S (古松), Fu YL (伏玉玲), Du MY (杜明远), Zhao L (赵亮), Zhao XQ (赵 新全), Yu GR (于贵瑞) (2004). Intergrated monitoring of Alpine vegetation types and its primary production. Acta Geographic Sinica (地理学报), 59, 40-48. (in Chinese with English abstract)

Liu YL (刘以连), Chou ZM (丑自明) (1994). Simulating model of growing processes of Leymus chinensis in meadow grassland in Inner Mongolia. Chinese Journal of Agrometeorology (中国农业 气象), 15(2), 43-46. (in Chinese)

Liu ZQ (刘振乾), Wang JW (王建武), Luo SM (骆世明), Lï XG (吕宪国), Liu ZL (刘兆礼), Liu HY (刘红玉), Li XJ (李秀军) (2002). On the safety threshold of wetlands based on water ecological element taking wetlands in Sanjiang Plain as an example. Chinese Journal of Applied Ecology (应用生态学报), 13, 1610 - 1614. (in Chinese with English abstract)

Ma KP (马克平) (1993). Seasonal models of the litter dynamics in Calamagrostis angustifolia grassland. Grassland of China (中 
国草地)，(5), 56-59. (in Chinese with English abstract) Ma KP (马克平) (1995). Studies on the structure and function of Calamagrostis angustifolia grassland ecosystem: energy fixation and allocation. Acta Ecologica Sinica (生态学报), 15, 23 31. (in Chinese with English abstract)

Ma KP (马克平), Zhou RC (周瑞昌), Zhang Y (张悦) (1993). Studies on the structures and seasonal dynamics of aboveground biomass of Calamagrostis angustifolia grassland in Sanjiang Plain. Grassland of China (中国草地), (2), 27 31. (in Chinese with English abstract)

Melillo JM, McGuire AD, Kicklighter DW, Moore B, Vorosmarty CJ (1993). Global climate change and terrestrial net primary production. Nature, 363, $234-240$.

Ni HW (倪红伟) (1996). Studies on the biomass of aboveground organs and layer structure of Deyeuxia angustifolia in typical meadow on Sanjiang Plain. Bulletin of Botanical Research (植物 研究), 16, 356-362. (in Chinese with English abstract)

Ni HW (倪红伟), Gao YK (高亦珂), Nie JL（聂江力） (1999). Analysis on the dynamic of aboveground biomass increment and time sequence of different Deyeuxia angustifolia populations. Bulletin of Botanical Research (植物研究), 19, 220 226. (in Chinese with English abstract)

Ni HW (倪红伟), Zhang X (张兴), Jia L (贾利), Gao YH (高 玉慧)，Wu HY (吴海一) (1998). The aboveground biomass dynamic of Deyeuxia angustifolia populations in typical meadow on Sanjiang Plain. Bulletin of Botanical Research (植物研究), 8, 328 - 335. (in Chinese with English abstract)

Qu Y (曲颖), Li ZZ (李自珍), Li WL (李文龙) (2004).

Amelioration on a growth model of plants in a wetland and dynamic computer simulation and analysis. Acta Botanica Boreali-Occidentalia Sinica (西北植物学报), 24, 418 - 423 . (in Chinese with English abstract)

Rasse DP, Stolaki S, Peresta G, Drake BG (2002). Patterns of canopy-air $\mathrm{CO}_{2}$ concentration in a brackish wetland: analysis of a decade of measurements and the simulated effects on the vegetation. Agricultural and Forest Meteorology, 114, 59- 73 .

Richert M, Saarnio S, Juutinen S, Silvola J, Augustin J, Merbach W (2000). Distribution of assimilated carbon in the system Phragmites australis-waterlogged peat soil after carbon-14 pulse labeling. Biological Fertility of Soils, 32, 1-7.

Shukla VP (1998). Modelling the dynamics of wetland macrophytes: Keoladeo National Park wetland, India. Ecological Modelling, 109, $99-114$.

Sorrell BK, Mendelssohn IA, Mckee KL, and Woods RA (2000). Ecophysiology of wetland plant roots: a modelling comparison of aeration in relation to species distribution. Annals of Botany, 86, $675-685$.
Sturtevant BR (1998). A model of wetland vegetation dynamics in simulated beaver impoundments. Ecological Modelling, 112, 195 -225 .

Sun XL (孙雪利), Liu JS (刘景双), Zhu YR (褚衍儒) (2000). Nitrogen dynamic in different organs of Calamagrostis angustifolia and Carex lasiocarpa in Sanjiang Plain. Chinese Journal of Applied Ecology (应用生态学报)，11, 893-897

Tong CL (童成立), Zhang WJ (张文菊), Tang Y (汤阳), Wang HQ (王洪庆) (2005). Estimation of daily solar radiation in China. Chinese Journal of Agrometeorology (中国农业气 象), 26, 165 - 169. (in Chinese with English abstract)

Woodward FI, Smith TM, Emanuel WR (1995) . A global land primary productivity and phytogeography model. Global Biogeochemical Cycles, 9, $471-490$.

Yan XD (延晓冬), Zhao SD (赵士洞), Yu ZL (于振良) (2000). Modeling growth and succession of northeastern China forests and its application in global change studies. Acta Phytoecologica Sinica (植物生态学报), 24, 1-8. (in Chinese with English abstract)

Yang YX (杨永兴), Wang SY (王世岩), He TR (何太蓉), Tian K (田昆), Yang B (杨波) (2002). Studies on plant biomass and its seasonal dynamics of typical wetland ecosystem in Sanjiang Plain. Grassland of China (中国草地), 24(1), 17. (in Chinese with English abstract)

Yang ZF (杨兆芳), Yu YQ (于永强), Huang Y (黄耀) $(2005)$. Simulating net primary production of rice and wheat crops: model validation and scenario prediction. Environmental Science (环境科学), 26(2), 16-20. (in Chinese with English abstract)

Yu M (喻梅), Gao Q (高琼), Gao SH (高素华) (1997). An ecophysiological model for individual plant under global change. Acta Botanica Sinica (植物学报), 39, 811-830. (in Chinese with English abstract)

Zhang Y, Li C, Zhou X, Moor B III (2002). A simulation model linking crop growth and soil biogeochemistry for sustainable agriculture. Ecological Modelling, 151, 75 - 108.

Zhou GS (周广胜), Zhang XS (张新时) (1996). Study on NPP of natural vegetation in China under global climate change. Acta Phytoecologica Sinica (植物生态学报), 20,11-19. (in Chinese with English abstract)

Zhou YH (周允华) (1990). Advance in PAR research. In: Beijing Agriculture Ecosystem Experimont Station of the Chinese Academy of Sciences (中国科学院北京农业生态系统试验 站）ed. Studies on Environmental Experiment of Agriculture Crops (农田作物环境实验研究). China Meteorological Press, Beijing. (in Chinese) 